\title{
Clinical Reasoning An Exercise in Clinical Reasoning: Do You Unpack?
}

\author{
Stacey Watkins, MD, PhD ${ }^{7}$, Angelo L. Gaffo, MD, MPSH ${ }^{2,3}$, Amanda V. Clark, MD 4 , and \\ Starr Steinhilber, $M D, M P H^{2,3}$
}

\begin{abstract}
${ }^{1}$ Tinsley Harrison Internal Medicine Residency Program, University of Alabama at Birmingham, Birmingham, AL, USA; 'irmingham Veterans Affairs Medical Center, Birmingham, AL, USA; ${ }^{3}$ University of Alabama at Birmingham, Birmingham, AL, USA; ${ }^{4}$ University of Mississippi Medical Center, Jackson, MS, USA.
\end{abstract}

KEY WORDS: clinical reasoning; unpacking principle; oral ulcers; uveitis.

J Gen Intern Med 33(7):1196-200

DOI: $10.1007 / \mathrm{s} 11606-018-4466-\mathrm{y}$

(C) Society of General Internal Medicine 2018

I $\mathrm{n}$ this series, a clinician extemporaneously discusses the diagnostic approach (regular text) to sequentially presented clinical information (bold). Additional commentary on the diagnostic reasoning process (italics) is integrated throughout the discussion.

A 27-year-old African-American man presented to the emergency department (ED) with five months of recurrent, sharp, left-sided chest pain with radiation to the back. The pain was worse with coughing and lying flat and was associated with shortness of breath. He had multiple normal evaluations in local EDs over recent months for identical symptoms that included electrocardiograms (ECGs), chest $x$-rays, troponins, and basic laboratory studies.

These symptoms are concerning and immediately create a broad differential diagnosis as it is uncommon for this age group to have chest pain at all, much less months of recurrent chest pain. This demographic often has costochondritis and other types of musculoskeletal pain rather than acute coronary syndrome or atherosclerotic disease. Congenital cardiac conditions and conditions related to drugs, specifically cocaine, should also be considered. Given the recurrent nature of his symptoms over the course of multiple months as well as the previous normal evaluations, costochondritis and musculoskeletal pain are also less likely but the differential remains wide with this limited information.

The clinician begins by stating the most commonly encountered diagnoses for chest pain in this demographic. This initial differential diagnosis is influenced by knowledge of the prevalence and incidence of particular disease. However, knowledge that this patient has had multiple prior unremarkable evaluations likely changes the clinician's perceived probability of common diseases when formulating the differential diagnosis. Given repeated prior evaluations in other EDs, he/she likely assumes that life-threatening causes have been ruled

Published online May 9, 2018 out. Considering this patient's condition has yet to be identified through repeated basic evaluations, the clinician recognizes the importance of maintaining a broad list of possible diagnoses.

Review of systems revealed a five-month history of headaches (bitemporal, pressure-like, non-radiating) and personality changes. His baseline mood was jovial and outgoing; however, his affect had become flat with anhedonia. Family reported intermittent episodes of decreased alertness. Additionally, he reported intermittent bilateral knee and elbow joint pain without swelling, erythema, or trauma.

These multiple symptom complaints raise concern for a syndrome rather than an exclusively organ-based condition. Headaches can be a consequence of vascular conditions affecting the blood supply with a differential of stroke, migraine, migraine-equivalent, or vascular inflammatory disorder. Personality changes with decreased alertness suggest an intrinsic brain parenchymal process. These symptoms are recurrent and intermittent, which could be seen with posterior basal ganglia involvement. Additionally, the intermittent joint pains without swelling could reflect an early inflammatory arthritis. The most common inflammatory disorders in this age group are systemic lupus erythematous (SLE), seronegative spondyloarthropathies, and juvenile arthritis and should be considered at this time.

When faced with further data, the clinician uses the "unpacking principle," which states that a more detailed description of an implicit hypothesis generally increases its judged probability. ${ }^{1,2}$ One adaptation of the unpacking principle to clinical practice is as a complaint becomes more specific and detailed, the likelihood of relevant and correct hypotheses being triggered increases. ${ }^{3,4}$ For example, myocardial infarction, pleuritis, or pericarditis is more likely to be considered when a clinician unpacks the differential of "chest pain" rather than keeping in mind only the broad category of "cardiac." Similarly, delineating "specific" complaints in the review of systems-bitemporal headaches, mood changes, decreased alertness, and arthralgias - helps the clinician "unpack" further diagnostic possibilities. ${ }^{3,4}$

Direct questioning and an extensive review of systems sometimes elicits the detailed description required for a diagnosis, although there is literature to suggest that more data is not necessarily better for clinical decision-making as it can 
lead to information overload. The unpacking principle is one way in which more "specific" and detailed patient history facilitates a more complete range of diagnostic possibilities. ${ }^{3,4}$

Upon review of past history, this patient was seen at the same hospital over one year ago for blurry vision and floaters in his left eye with accompanied headaches and was diagnosed with left eye anterior uveitis. Examination of his right eye at that time revealed a shrunken, phthisical eye that had no light perception. This was believed to be secondary to chronic uveitis and retinal detachment. Subsequently, the patient was lost to follow-up. No other significant past medical or surgical history was described. The patient did not have any alcohol, tobacco, or illicit drug use and no other high-risk behavior.

Summarizing everything thus far, this is a 27 -year-old African-American man with a past history of uveitis that presents with subacute chest pain, intermittent joint pains, and headache with personality changes suggesting a systemic process with cardiovascular and central nervous system (CNS) components. The differential is still broad and includes malignancy, infectious processes, and rheumatologic disorders. It would be important to rule out lymphoma and metastatic disease to the eye (for example, melanoma). Thinking about infectious etiologies, the term "phthiscal" is the ancient description of tuberculosis which certainly can involve multiple organs; however, involvement in the CNS is often more acute in presentation compared to the time course of this patient. Other important diseases to consider when formulating an infectious differential include syphilis, candidiasis, and histoplasmosis. One cannot help but focus on this clearly abnormal eye and wonder how it relates to his complaints, such as recurrent chest pain.

Looking at possible inflammatory processes, there are multiple conditions to consider. Juvenile arthritis can present with uveitis and joint pain. Seronegative spondyloarthropathies, including ankylosing spondylytis (AS), psoriatic arthritis, reactive arthritis, and arthritis associated with inflammatory bowel disease (IBD) can do this as well though some are less common in African Americans. Most of these can present with uveitis but they would be unusual to cause CNS events. Certainly, SLE has to be considered in this context, though SLE more commonly causes dry eyes and scleritis rather than uveitis. It can affect the CNS and also the cardiovascular system, specifically causing pericarditis and myocarditis. Sarcoidosis must be included in the differential given its prevalence among American Americans as well as its common ocular, cardiac, and neurologic involvements. We generally think of sarcoidosis as affecting primarily the lung but it can present with extrapulmonary disease in some patients including joint, cardiac, and skin manifestations similar to those in this patient. Lastly, Behçet's syndrome similarly affects young adults and can cause eye hypopyons, which can progress to uveitis. Behçet's syndrome causes joint pains and thrombosis, which could tie to the complaint of chest pain. All of these diseases should be considered based on the information available at this time.

At this point, the clinician takes a step back to summarize the large amount of clinical data. The clinician begins by stating the problem representation (PR), which is the "oneliner" or statement summarizing the key features of the presentation, usually in abstract terms. ${ }^{5,6}$ After stating the PR, the clinician then "unpacks" the broad categories of malignancy, infection, and inflammatory disorders. By using the most specific clinical highlights of the case-subacute chest pain, intermittent joint pains, and headache with personality changes-the clinician is able to further generate the full range of diagnostic possibilities.

On physical examination, the patient had normal vital signs. He was somnolent but arousable and fully oriented. His speech was mildly dysarthric without other deficits. He had normal muscle bulk, tone, and strength, intact sensation, and no involuntary movements or fasciculations. He had decreased frequency and amplitude of rapid alternating movements, left-sided dysmetria, an ataxic and broadbased gait, and a positive bilateral Romberg test. His reflexes were 3+ bilaterally with $3-4$ beat clonus in the right ankle, sustained clonus in the left ankle, bilateral Hoffman's signs, and Babinski responses were present. Skin exam highlighted multiple $2-4 \mathrm{~mm}$ oral mucosal ulcers and painless scrotal pustules. His eye exam revealed a left pupil that is round and reactive and 20/30 visual acuity, and slit-lamp examination was concerning for anterior and intermediate uveitis. The right eye was abnormal on inspection, and was described as shrunken and phthisical with no light perception (Fig. 1). Cranial nerves III-XII were normal (except limited movement of the right eye). He had no significant findings on musculoskeletal, cardiac, or pulmonary exam.

Most interestingly, the patient had oral and scrotal ulcers. The combination of oral and genital ulcers with uveitis and a CNS syndrome in a young male is very supportive of the

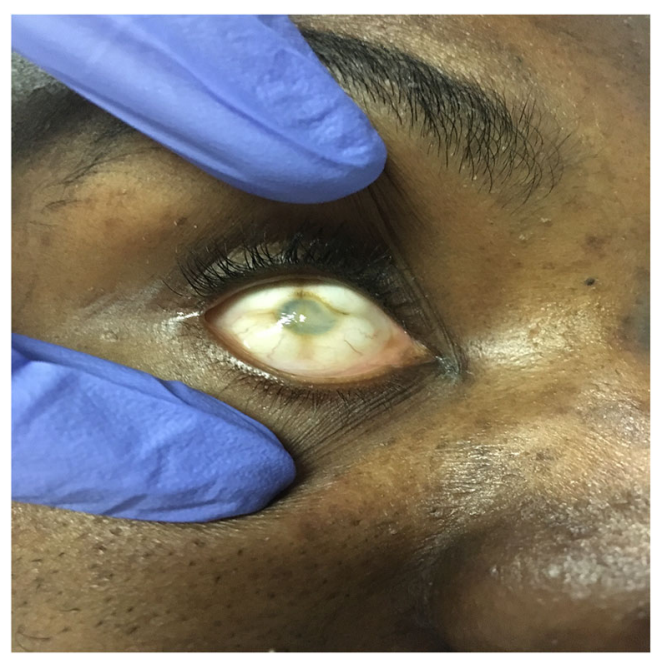

Figure 1 Patient's right eye. 
diagnosis of Behçet's syndrome. Ninety-five percent of patients with Behçet's syndrome will have painful oral ulcers. Genital ulcers, also common in Behçet's syndrome, are usually painful. While painful ulcerations are frequently reported in Behçet's syndrome, other very common skin manifestations include an acne-like rash or folliculitis, which can go unnoticed by patients when other cutaneous manifestations are present. It appears this patient has several manifestations previously described in patients with Behçet's syndrome.

Reactive arthritis secondary to gonorrhea seems less likely as it does not often cause ulcerations. Herpes virus (HSV) does cause ulcerations, though often painful, and can cause encephalitis. It traditionally presents with temporal lobe involvement, which would be seen on neuroimaging. Joint pains and his cardiovascular presentation would be difficult to unify with herpes. Often, SLE, sarcoidosis, and syphilis are considered when a constellation of symptoms is presented as in this case. SLE and sarcoidosis can cause ulcerations and have been known to have genital and mucosal ulcerations that can be painful or painless. Syphilis can present with painless genital lesions, oral ulcerations, and CNS findings. Lastly, we cannot exclude malignancy (for example, lymphoma and metastases), HIV-related diseases, or cerebral abscesses at this time.

The clinician is now able to use the physical exam findings to correlate with his initial differential diagnosis. He operates by pattern recognition here and realizes that many of these symptoms match his illness script for Behçet's syndrome. Illness scripts are used unconsciously by physicians when they assess and diagnose a patient. Illness scripts can contain details of pathophysiology, epidemiology, signs, symptoms, time course, diagnostics, and treatment and are often based on real patient experiences. ${ }^{5,7}$ Because experience plays a large role in formulating illness scripts, expert clinicians often have and enlist more illness scripts than novice learners. Utilizing illness scripts allows one to keep a "rolodex" of key features of diseases without committing extensive specifics to memory. ${ }^{8,9}$ They contain extensive clinically relevant information about how diseases present; in other words, what does a certain disease "look like?" A potential illness script for Behçet's syndrome for this clinician may be younger male patient, oral and genital ulcers, uveitis, and neurologic symptoms.

Basic laboratory studies demonstrated a mild microcytic anemia and a creatinine of $1.2 \mathrm{mg} / \mathrm{dL}$. He had an elevated C-reactive protein (CRP) at $78 \mathrm{mg} / \mathrm{L}$ and erythrocyte sedimentation rate (ESR) at $25 \mathrm{~mm} / \mathrm{h}$. Troponin and chest radiograph were unremarkable. The ECG showed diffuse ST segment elevations and PR segment depressions in the majority of limb and precordial leads. Transthoracic echocardiogram was unremarkable.

After a normal non-contrast head computed tomography, a traumatic lumbar puncture (LP) revealed cerebrospinal fluid (CSF) protein of $48 \mathrm{mg} / \mathrm{dL}$, glucose of $57 \mathrm{mg} /$ dL, 1150 red blood cells per cubic millimeter of blood, and 25 white blood cells per cubic millimeter of blood (5\% neutrophils and $77 \%$ lymphocytes). The opening pressure was not obtained.

The ECG is consistent with pericarditis and the elevated inflammatory markers reinforce the presence of systemic inflammation but are not specific enough to make a diagnosis. Lymphocyte predominant pleocytosis in CSF is also nonspecific and can be induced by many of the conditions we have discussed, including Behçet's, lymphoma, SLE, syphilis, sarcoidosis, or herpes virus. The LP is most useful to rule out herpes encephalitis and syphilis through serologic testing. If one wanted to treat for a rheumatologic cause with immunosuppressants, it is very important to await the results of these tests. Sarcoidosis is less likely given unremarkable chest radiograph but I would also check serum and CSF angiotensinconverting enzyme (ACE) levels. Marked elevation of these laboratory values would support a diagnosis of sarcoidosis. ${ }^{10} \mathrm{I}$ am very interested in a magnetic resonance image (MRI) of the brain as the patient seems to clinically have Behcet's syndrome, which has characteristic MRI findings. I would specifically inform the radiologist that Neuro-Behçet's is of high likelihood in this patient and to carefully consider this diagnosis as he/she is analyzing this patient's images.

The clinician has decided that the patient's clinical presentation and data obtained thus far is most consistent with the diagnosis of Behçet's syndrome and requests a test (MRI) that will confirm his/her clinical suspicion. The order for the MRI included the comment "evaluate for Neuro-Behçet's." Unpacking the broad categories into specific possibilities can remind the radiologist of possibilities they may otherwise overlook and increase the perceived likelihood of a disease. ${ }^{3,4}$ The providers are building a case for their presumed diagnosis by being increasingly detailed and specific, which may increase the likelihood of recognition of a specific and rare disease.

An MRI of the brain showed multifocal T2/FLAIR hyperintense lesions involving the left middle cerebellar peduncle and midbrain (bilateral, right greater than left) (Fig. 2), with extension from the right midbrain along the cerebral peduncle into the thalamus and internal capsule. Multifocal hypodensities were noted in bilateral basal ganglia with minimal extension to the periventricular white matter (Fig. 2).

Comprehensive infectious and rheumatologic panels were unremarkable (Table 1).

Some of our initial assumptions about the neuroanatomical location of the lesions are confirmed by imaging. The involvement of the thalamus, basal ganglia, and brainstem are consistent with Behçet's syndrome. The imaging does not support a primary demyelinating disorder, syphilis, or spondyloarthropathies from reactive arthritis, AS, or IBD. The lack of temporal lobe involvement and CSF studies makes HSV less likely. CNS involvement in SLE can be very diffuse, although is usually demyelinating, and is not often in this distribution. 

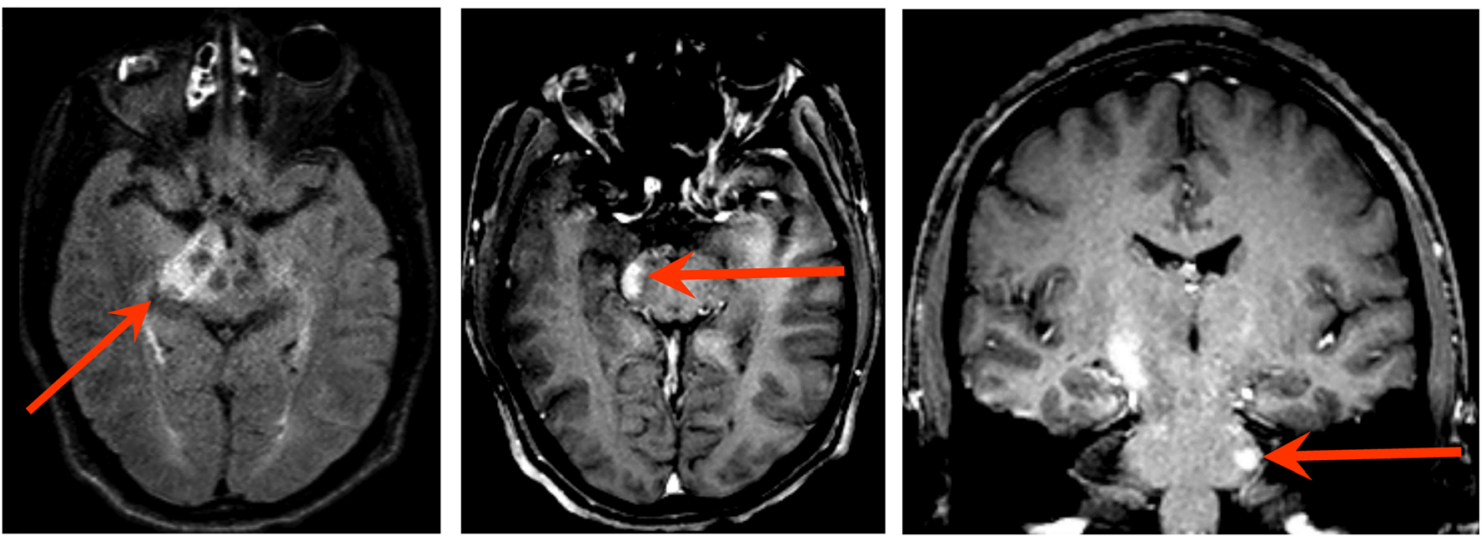

Figure 2 Magnetic resonance imaging of the patient's brain. a Axial T2/Flair MRI section highlighting abnormalities involving the right mesodiencephalic junction with patchy areas of enhancement (red arrow). b Axial T1 MRI section with hyperintensity present in the midbrain (red arrow). c Coronal T1 MRI section with hyperintensity present in the left cerebral peduncle (red arrow).

Combining the imaging with the extensive negative rheumatologic and infectious work-up presented, I favor a diagnosis of Behçet's syndrome. It is important to consider empiric treatment for the most likely and potentially serious conditions. Because of this, I would treat the patient with high-dose glucocorticoids.

A clinical diagnosis of Neuro-Behçet's disease (NBD) was made, and he received a five-day course of high-dose intravenous (IV) methylprednisolone. His neurologic symptoms improved drastically and his personality returned to near baseline. His speech became clear and coherent, dysmetria resolved, and his bilateral ankle clonus improved. He transitioned to oral prednisone and azathioprine on discharge and was seen in clinic two weeks later with no obvious neurological deficits and normalization of his CRP and ESR.

\section{DISCUSSION}

In this case, we highlight the use of the unpacking principle, which states that a more detailed description of an implicit hypothesis generally increases its judged probability. ${ }^{1,2}$ By analogy, the elicitation of patient-specific signs, symptoms, laboratory, or radiographic results may help clinicians consider the full range of possibilities of diagnosis and correctly judge their probabilities. This "unpacking" of specific information can be used as a cognitive strategy to facilitate the clinician's approach to his or her decision-making. ${ }^{3,4}$

When assembling a detailed data set in order to trigger additional diagnostic possibilities, there is a risk of becoming overloaded by information and contending with an overly exhaustive differential diagnosis. In this case, the clinician needed the more detailed history of bitemporal headaches, mood changes, and decreased alertness, to access larger categories of diagnostic possibilities. The clinician selected specific signs (oral ulcers, uveitis), symptoms (neurologic and mood changes), laboratory tests (CSF pleocytosis), and radiology (MRI findings) to make the correct diagnosis, while simultaneously not unpacking less specific issues such as elevated inflammatory markers, arthralgias, and nonspecific chest pain. This enabled this experienced clinician to broaden the differential diagnosis without getting overwhelmed by unpacking every detail of the case.

\section{CLINICAL TEACHING POINTS}

1. Behçet's syndrome is a rare multisystem chronic inflammatory disease, which preferentially affects young adults. It can affect many organ systems but is classically characterized by aphthous oral ulcers, genital ulcers, and uveitis and has a strong predisposition to cause thrombosis. Behçet's syndrome is also associated with multiple cardiac abnormalities including intracardiac thrombi, myocarditis, pericarditis, myocardial infarction, and valvular disease. ${ }^{11}$

2. Neurological manifestations of Behçet's syndrome, known as Neuro-Behçet's disease, are rare, occurring in less than $10 \%$ of Behçet's patients, but potentially devastating clinically. NBD is classified into two main disease groups: parenchymal and non-parenchymal. ${ }^{12}$ Parenchymal NBD, as described in this case, is subacute and focuses on involvement of focal or diffuse areas of the brain parenchyma, which are easily identified on MRI. Ocular involvement is included in parenchymal NBD. Nonparenchymal NBD is believed to be a neurovascular disease that affects the main vascular structures of the central nervous system, and the clinical presentation correlates to the location of vasculature involved. ${ }^{12}$ Significant morbidity is associated with ocular involvement in Behçet's 
Table 1 Results of Infectious and Rheumatologic Laboratory Studies

\begin{tabular}{|c|c|c|c|}
\hline$\overline{\text { ANA }}$ & $<1: 80$ & Blood cultures & Negative \\
\hline Anti-DNA & $<1: 10$ & T-SPOT & Negative \\
\hline Anti-SSA/Ro & $<12$ units & HIV Ab & Negative \\
\hline Anti-SSB/La & $<12$ units & $\begin{array}{l}\text { Toxoplasmosis } \\
\text { IgM }\end{array}$ & $\begin{array}{l}\text { Non- } \\
\text { reactive }\end{array}$ \\
\hline Anti-RNP & $<12$ units & $\begin{array}{l}\text { Toxoplasmosis } \\
\text { IgG }\end{array}$ & $\begin{array}{l}\text { Non- } \\
\text { reactive }\end{array}$ \\
\hline Anti-Smith & $<12$ units & EBV capsid IgM & Negative \\
\hline C-ANCA & $<1: 20$ & EBV capsid IgG & Positive \\
\hline P-ANCA & $<1: 20$ & CMV IgM & Negative \\
\hline $\begin{array}{l}\text { Anti-cardiolipin } \\
\text { IgM }\end{array}$ & $<9$ units $/ \mathrm{mL}$ & CMV Ab & Negative \\
\hline $\begin{array}{l}\text { Anti-cardiolipin } \\
\text { IgG }\end{array}$ & $<9$ units $/ \mathrm{mL}$ & CSF HSV PCR & Negative \\
\hline $\begin{array}{l}\text { Beta2- } \\
\text { glycoprotein I } \\
\text { IgM }\end{array}$ & $<9$ units & $\begin{array}{l}\text { CSF parvovirus } \\
\text { PCR }\end{array}$ & Negative \\
\hline $\begin{array}{l}\text { Beta2- } \\
\text { glycoprotein I } \\
\text { IgG }\end{array}$ & $<9$ units & CSF VDRL & Negative \\
\hline $\begin{array}{l}\text { Lupus } \\
\text { anticoagulant }\end{array}$ & Negative & Urine GC RNA & Negative \\
\hline Serum ACE & $\begin{array}{l}25 \text { units/L (9- } \\
67 \text { units/L) }\end{array}$ & $\begin{array}{l}\text { Urine chlamydia } \\
\text { RNA }\end{array}$ & Negative \\
\hline \multirow[t]{2}{*}{ CSF ACE } & $\begin{array}{l}8 \text { units } / \mathrm{L}(< \\
15 \text { units/L) }\end{array}$ & Treponema Ab & Negative \\
\hline & & $\begin{array}{l}\text { Scrotal ulcer } \\
\text { HSV PCR }\end{array}$ & Negative \\
\hline Kappa FLC & $\begin{array}{l}10.6 \mathrm{mg} / \mathrm{L} \\
(3.3-19.6 \mathrm{mg} / \\
\mathrm{L})\end{array}$ & $\begin{array}{l}\text { Oral ulcer HSV } \\
\text { PCR }\end{array}$ & Negative \\
\hline Lambda FLC & $\begin{array}{l}11.8 \mathrm{mg} / \mathrm{L} \\
(5.7-26.3 \mathrm{mg} / \\
\mathrm{L})\end{array}$ & & \\
\hline FLC ratio & $\begin{array}{l}0.9(0.26- \\
1.65)\end{array}$ & & \\
\hline \multicolumn{4}{|l|}{ SPEP } \\
\hline Total protein & $\begin{array}{l}7.0 \mathrm{~g} / \mathrm{dL}(6.0- \\
7.9 \mathrm{~g} / \mathrm{dL})\end{array}$ & & \\
\hline Albumin & $\begin{array}{l}3.3 \mathrm{~g} / \mathrm{dL} \\
(3.31-5.45 \mathrm{~g} / \\
\mathrm{dL})\end{array}$ & & \\
\hline Alpha1 & $\begin{array}{l}0.48 \mathrm{~g} / \mathrm{dL} \\
(0.19-0.46 \mathrm{~g} / \\
\mathrm{dL})\end{array}$ & & \\
\hline Alpha 2 & $\begin{array}{l}1.02 \mathrm{~g} / \mathrm{dL} \\
(0.48-1.05 \mathrm{~g} / \\
\mathrm{dL})\end{array}$ & & \\
\hline Beta & $\begin{array}{l}0.92 \mathrm{~g} / \mathrm{dL} \\
(0.48-1.10 \mathrm{~g} / \\
\mathrm{dL})\end{array}$ & & \\
\hline Gamma & $\begin{array}{l}1.29 \mathrm{~g} / \mathrm{dL} \\
(0.62-1.51 \mathrm{~g} / \\
\mathrm{dL})\end{array}$ & & \\
\hline
\end{tabular}

Reference ranges in parenthesis when appropriate

ANA, anti-nuclear antibody; Anti-DNA, anti-deoxyribonucleic acid antibody; Anti-SSA/Ro, anti-Sjogren's syndrome A antibody; Anti-SSB/ $L a$, anti-Sjogren's syndrome $B$ antibody; Anti-RNP, antiribonucleoprotein antibody; Anti-Smith, anti-Smith antibody; C-ANCA, cytoplasmic anti-neutrophil cytoplasmic antibodies; P-ANCA, perinuclear anti-neutrophil cytoplasmic antibodies; IgM, immunoglobulin $M$; IgG, immunoglobulin $G$; ACE, angiotensin-converting enzyme; $C S F$, cerebrospinal fluid; FLC, free light chain; SPEP, serum protein electrophoresis; HIV, human immunodeficiency virus; Ab, Antibody; EBV, Epstein-Barr virus; CMV, cytomegalovirus; HSV, herpes simplex virus; VDRL, venereal disease research laboratory; GC, gonorrhea; $P C R$, polymerase chain reaction; $R N A$, ribonucleic acid; g, gram; $L=$ liter; mg, milligram; $d L$, deciliter

syndrome and NBD, but visual symptoms can improve with prompt treatment. ${ }^{13-15}$
3. Initial therapy for parenchymal NBD is high-dose intravenous glucocorticoids (1 g IV methylprednisolone or equivalent) daily for 3-10 days followed by slow prednisone taper with close follow-up. ${ }^{12}$ Diseasemodifying therapy with azathioprine as first line is often added in patients based on severity, relapse, or other associated symptoms. $^{12}$

Acknowledgments: We thank Dr. Carlos A. Estrada for his advice regarding the manuscript.

Corresponding Author: Stacey Watkins, MD, PhD; Tinsley Harrison Internal Medicine Residency Program University of Alabama at Birmingham, Birmingham, AL, USA (e-mail: StaceyWatkins@uabmc. edu).

\section{Compliance with Ethical Standards:}

Conflict of Interest: The authors declare that they do not have a conflict of interest.

Disclosures: The opinions expressed in this article are those of the authors alone and do not reflect the views of the Department of Veterans Affairs.

\section{REFERENCES}

1. Tversky A, Koehler D. Support theory: a nonextensional representation of subjective probability. Psychol Rev. 1994;101(4):547-567.

2. Redelmeier D, Koehler D, Liberman V, Tversky A. Probability judgement in medicine: discounting unspecified possibilities. Med Decis Mak. 1995; 15(3):227-230.

3. Croskerry P. Achieving quality in clinical decision making: cognitive strategies and detection of bias. Acad Emerg Med. 2002;9(11):11841204. I

4. Croskerry $\mathbf{P}$. The importance of cognitive errors in diagnosis and strategies to minimize them. Acad Med. 2003;78(8):775-780.

5. Bowen JL. Educational strategies to promote clinical diagnostic reasoning. N Engl J Med. 2006;355(21):2217-2225.

6. Journal of General Internal Medicine. Problem Representation. Available at: http://www.sgim.org/web-only/clinical-reasoning-exercises/problem-representation-overview. Accessed 21 Mar 2018.

7. Journal of General Internal Medicine. Illness Scripts. Available at: http:// www.sgim.org/web-only/clinical-reasoning-exercises/illness-scriptsoverview. Accessed 21 Mar 2018.

8. Jones B, Brzezinski W, Estrada C, Rodriguez M, Kraemer R. A 22year-old woman with abdominal pain. J Gen Intern Med. 2014;7(29): 1074-1078

9. Charlin B, Boshuizen HP, Custers EJ, Feltovich PJ. Scripts and clinical reasoning. Med Educ. 2007;41(12):1178-1184.

10. Ungprasert P, Carmona E, Crowson C, Matteson E. Diagnostic utility of angiotensin-converting enzyme in sarcoidosis: a population-based study. Lung. 2016;194(1):91-95.

11. Demirelli S, Degirmenci H, Inci S, Arisoy A. Cardiac manifestations in Behcet's disease. Intractable Rare Dis Res. 2015;4(2):70-75.

12. Kalra S, Silman A, Akman-Demir G, et al. Diagnosis and management of Neuro-Behçet's disease: international consensus recommendations. J Neurol. 2014;261(9): 1662-1676.

13. Accorinti M, Pesci FR, Pirraglia MP, Abicca I, Pivetti-Pezzi P. Ocular Behçet's disease: changing patterns over time, complications and longterm visual prognosis. Ocul Immunol Inflamm. 2016:1-8.

14. Benezra D, Cohen E. Treatment and visual prognosis in Behçet's disease. Br J Ophthalmol. 1986;70:589-592.

15. Siva A, Kantarci OH, Altintas A, et al. Behçet's disease : diagnostic and prognostic aspects of neurological involvement. J Neurol. 2001;248:95103. 